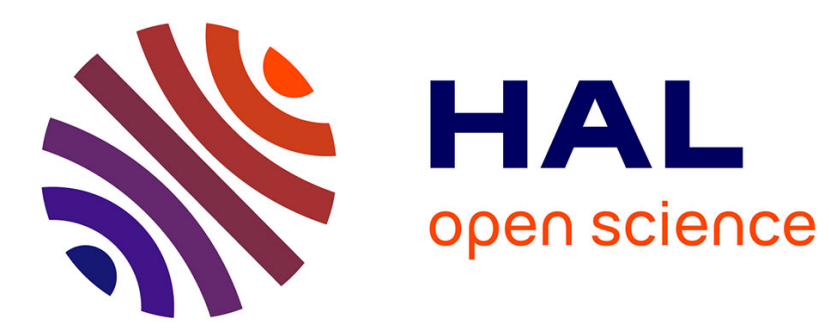

\title{
Non-Mean-Field Interpretation of the Irreversibility Line in Spin-Glasses
}

\author{
A P Malozemoff, S E Barnes, B. Barbara
}

\section{To cite this version:}

A P Malozemoff, S E Barnes, B. Barbara. Non-Mean-Field Interpretation of the Irreversibility Line in Spin-Glasses. Physical Review Letters, 1983, 51 (18), pp.1704. hal-01660009

\section{HAL Id: hal-01660009 \\ https://hal.science/hal-01660009}

Submitted on 9 Dec 2017

HAL is a multi-disciplinary open access archive for the deposit and dissemination of scientific research documents, whether they are published or not. The documents may come from teaching and research institutions in France or abroad, or from public or private research centers.
L'archive ouverte pluridisciplinaire HAL, est destinée au dépôt et à la diffusion de documents scientifiques de niveau recherche, publiés ou non, émanant des établissements d'enseignement et de recherche français ou étrangers, des laboratoires publics ou privés. 


\title{
Non-Mean-Field Interpretation of the Irreversibility Line in Spin-Glasses
}

\author{
A. P. Malozemoff \\ IBM Thomas J. Watson Research Center, Yorktown Heights, New York 10598 \\ and \\ S. E. Barnes \\ Physics Department, University of Miami, Coral Gables, Florida 33124
}

and

B. Barbara

Laboratoire Louis Neél, Centre National de la Recherche Scientifique, F-38042 Grenoble Cedex, France (Received 2 August 1983)

\begin{abstract}
Susceptibility data on an amorphous GdAl spin-glass show an irreversibility onset line $t=-A_{-} H^{2 / \phi_{-}}$and a susceptibility crossover line $t=+A_{+} H^{2 / \phi+}(t$ is reduced temperature; $H$ is field). The exponents $\phi$ are both close to 3 , and the amplitudes $A$ differ only by a factor of 2 . In contrast to earlier explanations of the irreversibility in terms of a de Almeida-Thouless mean-field instability, here both crossovers are explained in terms of a non-mean-field scaling theory.
\end{abstract}

PACS numbers: $75.30 . \mathrm{Kz}, 75.40 . \mathrm{Dy}, 75.50 . \mathrm{Kj}$

The mean-field theory of spin-glasses has a contradictory record of success and failure in comparison to experiment: success in predicting the $\frac{2}{3}$-power law for onset of irreversibility as a function of field below the transition temperature $T_{G}$, and failure in describing the scaling exponents of the susceptibility above $T_{G}$. Here we present a novel, physical interpretation of the irreversibility line based on a non-mean-field phase-transition model, which can account consistently for many aspects of the experimental data on an amorphous GdAl spin-glass both above and below $T_{G}$.

First we describe our data on the amorphous $\mathrm{Gd}_{37} \mathrm{Al}_{63}$ spin-glass. We have previously analyzed $^{1,2}$ the measured susceptibility $\chi$ above $T_{G}$ in terms of a scaling theory,

$$
\chi_{\text {sing }} \propto H^{2 / \delta} f_{+}\left(t / H^{2 / \phi}\right) \text {. }
$$

where $t=\left(T-T_{G}\right) / T_{G}$ is reduced temperature, $H$ is applied magnetic field, $\delta$ and $\phi$ are scaling exponents, and $f_{+}(x)$ is a scaling function which approaches a constant as $x \rightarrow 0$ and $x^{-\gamma}$ as $x \rightarrow \infty$, with the scaling relation $\gamma=\phi(\delta-1) / \delta$. The fit gave $\delta$ and $\phi_{+}$values of 5.7 and 3.3 , respectively, far from the mean-field values $\delta=\phi=2$. (Here $\phi_{+}$denotes the $t>0$ experimental value of the theoretical crossover exponent $\phi$.$) We also evaluat-$ ed the amplitude $A_{+}=3.3 \times 10^{-3} \mathrm{Oe}^{-2 / \phi}$ of the crossover line $t=A_{+} H^{2 / \phi_{+}}$which is implied by the argument of $f$ in Eq. (1). This line is the dashed line in Fig. 1. Scaling exponents have also been established in other spin-glass systems such as CuMn, ${ }^{3,4}$ and by and large the exponents come out far from the mean-field values and roughly similar to the ones given above, with some exceptions. ${ }^{5,6}$

Next we report new data on the irreversibility crossover line in our GdAl sample. As usual, ${ }^{7-11}$ the sample is cooled in an initial field $H_{i}$ below the transition temperature $T_{G}$ of $15.5 \mathrm{~K}$, and the field is changed to a new value $H_{f}$ which could be either larger or smaller than $H_{i}$. Conventionally $H_{i}=0$, and on heating in constant $H_{f}, \chi$ measured at a fixed time after successive temperature steps shows the well-known simple peak as a function of temperature. In Fig. 2 we show for variety the opposite case, with $H_{i}>H_{f}$. The data obtained on heating, shown as crosses, drop to a minimum, then rise to a cusp, and finally drop

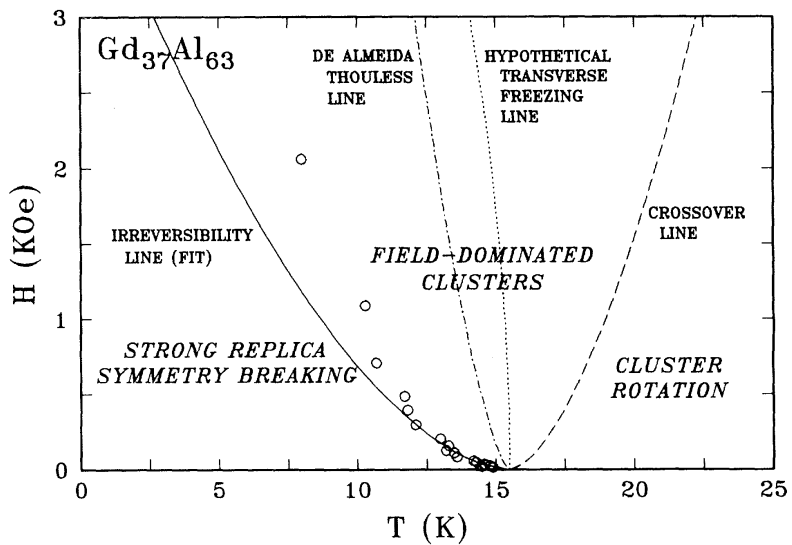

FIG. 1. Crossover and transition lines in the fieldtemperature plane for $\mathrm{Gd}_{37} \mathrm{Al}_{63}$ with $T_{G}(H=0)=15.5 \mathrm{~K}$. 


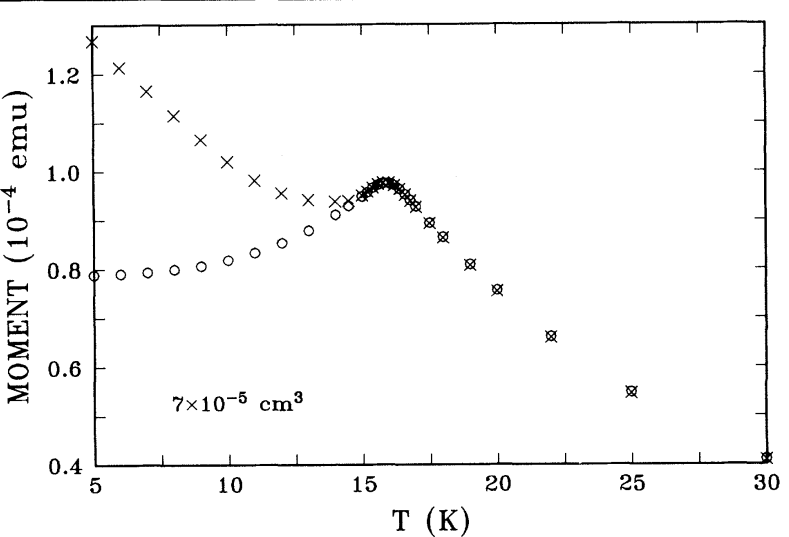

FIG. 2. Moment vs temperature for $\mathrm{Gd}_{37} \mathrm{Al}_{63}$. Crosses show data obtained after cooling to $5 \mathrm{~K}$ at 36.5 Oe and changing the field to $16.0 \mathrm{Oe}$, then increasing temperature at regular time intervals (about $5 \mathrm{~min}$ ). Circles show field-cooled data at 16.0 Oe. Note that the onset of irreversibility and the susceptibility peak do not coincide.

again. Data on cooling in the same field, shown as circles, are fully reversible and show the cusp but not in the low-temperature upturn. As usual, the difference between the two curves indicates irreversibility which appears to go to zero at about $14.9 \pm 0.1 \mathrm{~K}$ within experimental accuracy. Incidentally, the susceptibility cusp is clearly reversible, and hence its physics bears no obvious relationship to the irreversibility line, in contrast to the implication of Ising mean-field theory.

Although the irreversibility line may be measurement-time dependent, ${ }^{10}$ we see no significant shift within a decade change in measurement waiting times. We ascribe this behavior to the existence of two scales of relaxation times, a short one widely distributed and a long one relatively narrow. Therefore, we follow common practice in using the rough measure indicated above to determine it. In Fig. 1 we plot the irreversibility onset temperatures versus field, using data from $H_{i}>H_{f}$ and $H_{i}<H_{f}$ which are consistent within experimental error. A least-squares fit of data below about 300 Oe by $t=A_{-} H^{2 / \phi_{-}}$yields $\phi_{-}=3.5$, $A_{-}=8.2 \times 10^{-3} \mathrm{Oe}^{-2 / \phi_{-}}$, and the solid line in Fig. 1. Such results on the irreversibility line, showing an $\sim H^{2 / 3}$ dependence, have been obtained before in other systems. ${ }^{7-12}$ However, our results are the first to be obtained simultaneously with the high-temperature crossover analysis on a single sample. Remarkably, we find the powers $\phi_{+}$and $\phi$. to be essentially the same and the amplitudes to be only a factor of $2 \frac{1}{2}$ from each other.
Can theory predict the $\frac{2}{3}$ power? Recent computer simulations ${ }^{13,14}$ of nearest-neighbor twodimensional frustrated systems have shown $H^{2 / 3}$ relaxation behavior arising in the context of dynamic spin-glass freezing (i.e., no $T>0$ phase transition). However, the time and field scales as well as dimensionality of these studies makes their applicability to real systems still open to question. In what follows we consider models based on the assumption of a $T>0$ phase transition.

In a mean-field Ising model, de Almeida and Thouless ${ }^{15}$ and Sompolinsky ${ }^{16}$ (dATS) found replica-symmetry breaking along a line $t=-A H^{2 / 3}$, which corresponded physically to the limit of irreversibility in the spin-glass susceptibility $\chi$. In the extension of the theory to Heisenberg spins, ${ }^{17,18}$ replica symmetry is actually broken already at $t=0$ where transverse freezing occurs even in a field (except for $H^{2}$ corrections), but the dATS line can still be defined as a crossover from weak to strong irreversibility, since freezing of transverse degrees of freedom by itself is not expected to affect the longitudinal magnetization strongly. Comparison with experiment showed amplitudes $A$. typically an order of magnitude larger ${ }^{7-10}$ than predicted. In our case the predicted $A$ is $4.6 \times 10^{-4} \mathrm{Oe}^{-2 / 3}$, a factor of 18 smaller than our observed amplitude. ${ }^{19}$ Nevertheless, the successful prediction of the power law seemed encouraging for the mean-field theory.

This might seem quite satisfying if it were not that the crossover exponent $2 / \phi$ is unity within a mean-field model, implying crossover lines $t$ $\propto \pm H$. As we shall review below, there is a peculiar cancellation in the mean-field theory of a spin-glass that shifts the $t<0$ instability onto the $-H^{2 / 3}$ line. However, the $t>0$ crossover line is not affected. In this context, the tantalizing experimental similarity of $\phi_{-}$and $\phi_{+}$appears as a paradox, the former apparently agreeing with mean-field theory and the latter not.

An important clue to resolve this paradox comes from Bouchiat, ${ }^{20}$ who argued as follows: Within mean-field theory for an Ising glass, one has for negative $t\left(T<T_{G}\right)$ and to leading order in the field (i.e., for reduced field $h \ll|t|$ ),

$$
q=-t\left[1+\left(H^{2} / 2 t^{2}\right)\right]+O\left(t^{2}\right)
$$

while the Fischer formula, ${ }^{21}$ valid without replica-symmetry breaking, is

$$
M / H=(1-q) / T=(1-q) / T_{G}(1+t) .
$$

To lowest order these formulas are clearly com- 
patible with the non-mean-field (or scaling) model formula

$$
\chi=T^{-1}-t^{\beta} f_{-}\left(H^{2} / t^{\phi}\right)
$$

with $\phi=2$ and $\beta=1$. Here the term $T^{-1}$ represents the regular or noncritical part of $\chi$. But if one substitutes (2) into (3), one finds

$$
\begin{aligned}
M / H & =T_{G}{ }^{-1}\left[1-\left(H^{2} / 2 t\right)-\left(t^{2} / 3\right)+O\left(t^{3}\right)\right] \\
& \sim T_{G}{ }^{-1}-t^{2} f_{-}\left(H^{2} / t^{3}\right) .
\end{aligned}
$$

The term linear in $t$ has disappeared because the contribution of the regular term $T^{-1}$ cancels that of the singular term. Thus to lowest order the result looks like Eq. (4) but with $\phi=3$.

Normally one would expect ${ }^{22}$ any phase-transition line in the $H-t$ plane to be governed by the crossover exponent $\phi$, and so, from Eq. (4), one would expect the irreversibility line, if viewed as a phase-transition line, to occur along $t \sim-H$. However, Vannimenus, Thoulouse, and Parisi ${ }^{23}$ have compared the replica-symmetry-breaking line with the maximum in the susceptibility for Ising spin-glasses and have shown that the two coincide. They argue via the Maxwell relation $\partial S / \partial H=\partial M / \partial T$ that replica-symmetry breaking appears to occur in order to stop the entropy decreasing with decreasing field. Because of the cancellation, $d M / d T=0$ corresponds not to $t \propto-H$, but to $t \propto-H^{2 / 3}$, as one readily confirms from Eq. (5).

However, away from mean field, we must use the general scaling form of Eq. (4). Expanding both $T^{-1}$ and $f$, one has

$$
M / H=1-t-a|t|^{\beta}-b H^{2}|t|^{-\gamma},
$$

where $\gamma=\phi-\beta$. Because $\beta \neq 1$, the cancellation no longer takes place. Also both theoretical and experimental results suggest that $d M / d T=0$ does not correspond to replica-symmetry breaking for either a Heisenberg glass or reality. The obvious implication (contrary to Bouchiat's conclusion from the same facts) seems to us to be the following: In a non-mean-field model, strong replica-symmetry breaking and irreversibility should occur at the natural scaling crossover line $t \propto H^{2 / \phi_{-}}$, whereas in mean-field theory, a rather special cancellation of regular and singular terms suppresses the instability along this line, allowing the lower-temperature dATS instability to dominate. This simple hypothesis, coupled with the fact that in scaling theory $\phi_{+}=\phi_{-}$, allows us now to explain in a natural way the similarity of the experimentally observed irreversibility and high-temperature crossover exponents: If the system were mean field, the two lines would go as $-H^{2 / 3}$ and $+H$, respectively, but if it were non-mean-field, they would go as $-H^{2 / \phi}$ and $+H^{2 / \phi}$ with $\phi>2$, as observed.

Historically we can now see that two coincidences have obscured this simple picture up to now. The first is that the mean-field theory happens, because of the special cancellation, not to show an instability at the $t \propto-H$ line. The second is that the non-mean-field value of $\phi$ just happens to be close to 3 , giving a power $\frac{2}{3}$ which looks like that in the dATS theory.

To give further credence to our hypothesis that $t \propto-H^{2 / \phi}$ is an irreversibility line, we develop a physical picture of the spin-glass scaling theory introduced earlier, ${ }^{2}$ by considering regions or clusters of correlation length $\xi$ which diverges as $t^{-\nu}$ approaching $T_{G}$. These coherent regions have a volume $\xi^{d}$ where $d$ is dimensionality (possibly fractal if the clusters are branched). The regions have a magnetization $m_{\xi}$ which goes as $\xi^{d / 2}$ for a perfectly statistical distribution of spins in the cluster, or more generally $\xi^{d \phi}$ with the power $p$ expected to be near $\frac{1}{2}$. Defining $v d p$ as $\phi / 2$, we see that $m_{\xi} \propto t^{-\phi / 2}$ (at least in low field). Above $T_{G}$, the regions can be viewed as superparamagnetic clusters which rotate in response to the applied field, giving a net magnetization $N_{\xi} m_{\xi} \mathfrak{L}\left(m_{\xi} H / k T\right)$, where $N_{\xi}$ is the number of clusters and $\mathcal{L}$ is the Langevin function. ${ }^{4}$ The criterion $m_{\xi} H \propto k T$ then corresponds to a crossover for the clusters to align along the field. Since $m_{\xi} \propto t^{-\phi / 2}$, and $T$ is not critical, the crossover line $t \propto H^{2 / \phi}$ follows naturally. At higher fields, the susceptibility arises from field-induced spin flips within the cluster which are only weakly (noncritically) temperature dependent. Below $T_{G}$, the correlated regions of dimensions $\xi$ can be visualized as rotating in a frozen matrix. As with any magnetic phase transition, the matrix imposes internal energy barriers to rotation which are of order $k T \sim k T_{G}$, the motion being "driven" by the entropy; i.e., the terms $U$ and $S T$ of the free energy nearly cancel in $F=U-S T$. Thus, as a function of temperature, the barriers stay essentially fixed in average magnitude while the size of the clusters they refer to grows critically. In our picture, irreversibility, or perhaps more accurately very slow long-time behavior, arises because the barriers for thermal activation of correlation clusters are sufficiently high that the system drifts only slowly in search of its ground state among the many almost equiv- 
alent energy minima. Many correlated clusters must flip in each step and then be flipped again in some later step. But when a field is applied such that $m_{\xi} H>k T_{G}$, the cluster Zeeman energy begins to win out over the entropy to be gained through random orientation of $m_{\xi}$. Then the clusters flip relatively rapidly to point along the field and become essentially locked in that direction apart from the usual type of paramagnetic thermal fluctuations. Thus once again, as for $t>0$, the crossover condition is determined by $m_{\xi} H$, implying $t \propto-H^{2 / \phi}$. In fact, to the extent that scaling theory predicts a symmetric behavior for the correlation length above and below the phase transition, the exponent for the irreversibility crossover should equal that of the $t>0$ susceptibility crossover, and the amplitudes should be comparable, as observed experimentally (Fig. 1).

In summary, we can label the regions of the non-mean-field spin-glass phase diagram as in Fig. 1, using the simple physical notions described above. Although such diagrams have been presented before, both theoretically ${ }^{18}$ and experimentally, ${ }^{3,7}$ none of these have so far in our view identified all the physically relevant regions: a critical region for some range of $t>0$, a susceptibility crossover line $t \propto H^{2 / \phi}$, a transverse freezing at $t=0$ (with $H^{2}$ corrections), and an irreversibility crossover at $t \propto-H^{2 / \phi}$. In mean-field theory, the irreversibility crossover would shift to $t \propto-H^{2 / 3}$ (the dATS line), while the other crossover remains at $t \propto H^{2 / \phi}=H$, but we have shown that the non-mean-field version is, in fact, in much better agreement with experiment.

The authors appreciate conversations with H. Sompolinsky, E. Pytte, Y. Imry, and M. Fisher. This work was supported in part by the National Science Foundation-Solid State Physics Grant No. DMR 81-20827.

\footnotetext{
${ }^{1}$ B. Barbara, A. P. Malozemoff, and Y. Imry, Phys.
}

Rev. Lett. 47, 1852 (1981); A. P. Malozemoff, B. Barbara, and Y. Imry, J. Appl. Phys. 53, 2205 (1982).

${ }^{2} \mathrm{~B}$. Barbara and A. P. Malozemoff, to be published.

${ }^{3}$ A. Berton, J. Chaussy, J. Odin, R. Rammal, and R. Tournier, J. Phys. (Paris), Lett. 43, L153 (1982).

${ }^{4} \mathrm{R}$. Omari, thesis, University of Grenoble, 1982 (unpublished); R. Omari, J. J. Préjean, and J. Souletie, to be published.

${ }^{5}$ P. Monod and H. Bouchiat, J. Phys. (Paris), Lett. $\underline{43}$, L45 (1982). These authors report mean-field exponents, but we suspect that this is because they "force-fit" their data to the mean-field model rather than attempting a more general scaling fit.

${ }^{6}$ P. Beauvillain, C. Dupas, J. P. Renard, and P. Veillet, J. Magn. Magn. Mater. 31-34, 1377 (1983). These authors report non-mean-field exponents for $\delta$ and $\phi$ but a mean-field value for $\gamma$.

${ }^{7}$ R. V. Chamberlin, M. Hardiman, L. A. Turkevich, and R. Orbach, Phys. Rev. B 25, 6720 (1982).

${ }^{8} \mathrm{Y}$. Yeshurun and H. Sompolinsky, Phys. Rev. B $\underline{26}$, 1487 (1982).

${ }^{9}$ Y. Yeshurun, L. J. P. Ketelsen, and M. B. Salamon, Phys. Rev. B 26, 1491 (1982).

${ }^{10}$ N. Bontemps, J. Rajchenbach, and R. Orbach, J. Phys. (Paris), Lett. 44, L47 (1983).

${ }^{11}$ R. Wendler, K. Baberschke, P. Pureur, and A. Fert, Bull. Am. Phys. Soc. 28, 509 (1983); A. Fert, P. Pureur, F. Hippert, K. Baberschke, and F. Bruss, Phys. Rev. B 26, 5300 (1982).

${ }^{12}$ I. A. Campbell, D. Arvanitis, and A. Fert, Phys. Rev. Lett. 51, 57 (1983).

${ }^{13}$ A. P. Young, Phys. Rev. Lett. $\underline{50}, 917$ (1983).

${ }^{14}$ W. Kinzel and K. Binder, Phys. Rev. Lett. $\underline{50}, 1509$ (1983).

${ }^{15} \mathrm{~J}$. R. L. de Almeida and D. J. Thouless, J. Phys. A 11, 983 (1978).

${ }^{16} \mathrm{H}$. Sompolinsky, Phys. Rev. Lett. 47, 935 (1981).

${ }^{17}$ G. Toulouse and M. Gabay, J. Phys. (Paris), Lett. 42, L103 (1981).

${ }^{18}$ D. M. Cragg, D. Sherrington, and M. Gabay, Phys. Rev. Lett. 49 , 158 (1982); D. Elderfield and D. Sherrington, to be published.

${ }^{19} \mathrm{We}$ use the conventional Toulouse-Gabay formula (Ref. 17) without spin $S=\frac{7}{2}$, although the ElderfieldSherrington formula (Ref. 18) appears to differ and to require introduction of $S$.

${ }^{20} \mathrm{H}$. Bouchiat, J. Phys. C $\underline{16}$, L145 (1983).

${ }^{21}$ K. H. Fischer, Phys. Rev. Lett. 34, 1438 (1975).

${ }^{22}$ S. Singh and D. Jasnow, Phys. Rev. B 12, 493 (1975).

${ }^{23} \mathrm{~J}$. Vannimenus, G. Thoulouse, and G. Parisi, J. Phys. (Paris) 42, 565 (1981). 